\title{
Non-classical polar unitals in finite Figueroa planes
}

\author{
Man Wa Hui and Philip P. W. Wong
}

\begin{abstract}
The finite Figueroa planes are non-Desarguesian projective planes of order $q^{3}$ for all prime powers $q>2$. These planes were constructed algebraically in 1982 by Figueroa, and Hering and Schaeffer, and synthetically in 1986 by Grundhöfer. All Figueroa planes of finite square order are shown to possess a unitary polarity by de Resmini and Hamilton in 1998, and hence admit unitals. Using the result of O'Nan in 1971 on the non-existence of his configuration in a classical unital, and the intrinsic characterization by Taylor in 1974 of the notion of perpendicularity induced by a unitary polarity in the classical plane (introduced by Dembowski and Hughes in 1965), we show that these Figueroa polar unitals do not satisfy a necessary condition, introduced by Wilbrink in 1983, for a unitary block design to be classical, and hence they are not classical.
\end{abstract}

Mathematics Subject Classification (2010). 05B25, 51E05, 51A35.

Keywords. Figueroa plane, unitary polarity, Figueroa unital, classical unital, O'Nan configuration, Wilbrink's conditions.

\section{Introduction}

Let $U$ be a unital of order $n$, i.e. a (unitary block) design with parameters $2-\left(n^{3}+1, n+1,1\right)$ (see [3]). If $U$ is a subdesign of a projective plane $\pi$ of order $n^{2}$, i.e. a design with parameters $2-\left(n^{4}+n^{2}+1, n^{2}+1,1\right)$, then we call $U$ an embedded unital. If the points and lines of an embedded unital are respectively the absolute points and (restrictions of) non-absolute lines of a unitary polarity of the ambient plane, then we call $U$ a polar unital. The design defined by a polar unital for which the ambient plane is the classical plane $P G\left(2, n^{2}\right)$ is called a classical unital. The set of absolute points of a unitary polarity in

This work was partially supported by a grant from the Research Grant Council of the HKSAR, China (Project number: HKU7060/11P). 
$P G\left(2, n^{2}\right)$ is called a Hermitian curve (see [7,9]). A classical unital of order $n$ can therefore be embedded (as a polar unital) in the classical projective plane $P G\left(2, n^{2}\right)$. A natural question arises as to whether a classical unital can be embedded (as a polar unital) in a non-classical plane. At present this question seems to remain unanswered.

This article studies a specific example, namely the polar unital defined in 1998 by de Resmini and Hamilton [4] in the Figueroa plane. The Figueroa plane is a non-classical finite projective plane constructed algebraically in 1982 by Figueroa [5], Hering and Schaeffer [8], and synthetically in 1986 by Grundhöfer [6]. We shall refer to the polar unital under investigation as a Figueroa unital and denote it by $\mathcal{U}$. The unitary polarity defining $\mathcal{U}$ is induced by a unitary polarity in the classical plane $P G\left(2, q^{6}\right)$ defining a classical unital $\mathcal{H}$. We shall prove that $\mathcal{U}$ is non-classical by proving that it does not satisfy certain necessary condition for $\mathcal{H}$.

In 1983 Wilbrink [13] characterized the classical unital by three conditions, $(I),(I I)$ and $(I I I)$. We recall the conditions. Given a unital $U$ (of order $n$ ). An O'Nan configuration [10] in $U$ is a configuration of four distinct lines intersecting in six distinct points. Two lines missing a point $a$ are called $a$-parallel if they intersect the same lines through $a$. Wilbrink's conditions on $U$ are as follow:

Condition (I). O'Nan configuration does not exist.

Condition (II). Let a be a point, $L$ be a line through a, and $M$ be a line not incident with a such that $L$ meets $M$. For any point $b^{\prime}$ on $L$ which is different from a, there exists a line $M^{\prime}$ passing through $b^{\prime}$ but not a and intersecting all lines from a which meet $M$.

Condition (III). Let a be a point, $L_{1}, L_{2}$ and $L_{3}$ be three lines through a, and $b_{i}, c_{i}$ be points on $L_{i}(i=1,2,3)$. If $b_{1} \cdot b_{2}$ and $c_{1} \cdot c_{2}$ are a-parallel, and $b_{1} \cdot b_{3}$ and $c_{1} . c_{3}$ are a-parallel, then $b_{2} . b_{3}$ and $c_{2} . c_{3}$ are a-parallel.

We remark that condition $(I I)$ is a weak form of the notion of perpendicularity of Taylor [12]. We shall prove that $\mathcal{U}$ does not satisfy condition $(I I)$. The main idea is to make use of the deviation of $\mathcal{U}$ from $\mathcal{H}$ where some intersection properties of $\mathcal{H}$ will be lost in $\mathcal{U}$. After recalling the pertinent facts about the Figueroa plane and unital in Sects. 2 and 3, we prepare the technicalities in the form of various structure theorems to study these intersection properties in Sect. 4, and prove our main result in Sect. 5 .

\section{Finite Figueroa planes of square order}

Let $\alpha$ be an order 3 planar collineation of the classical projective plane $P G\left(2, q^{6}\right)$ of order $q^{6}$ over the finite field $G F\left(q^{6}\right)$, where the fixed elements of $\alpha$ constitute a subplane $\mathcal{P}_{\alpha}$ isomorphic to $P G\left(2, q^{2}\right)$. The points and lines of $P G\left(2, q^{6}\right)$ are classified into distinct types, as follows. A point $x$ of $P G\left(2, q^{6}\right)$ 
is said to be of type I if $x^{\alpha}=x$; or type II if $x, x^{\alpha}, x^{\alpha^{2}}$ are distinct and collinear; or type III if $x, x^{\alpha}, x^{\alpha^{2}}$ are distinct and noncollinear. Types of lines of $P G\left(2, q^{6}\right)$ are defined dually. Points and lines of type I thus constitute $\mathcal{P}_{\alpha}$. If $x$ is a type II point, then it is on the unique type I line $L=x \cdot x^{\alpha} \cdot x^{\alpha^{2}}$. Conversely, if a point is on a unique type I line, then it is of type II since a type I point is on $q^{2}+1$ type I lines and a type III point is on no type I line. It follows that if (and only if) a point is on no type I line then it is of type III. Simple counting then gives the pencil structures of points of different types. For ease of reference we summarize these observations in the following lemma (see also [5]):

Lemma 2.1. 1. A point is of type II if and only if it is on a unique type I line.

2. A point is of type III if and only if it is on no type I line.

3. The pencil of a type I point consists of $q^{2}+1$ type I lines and $q^{6}-q^{2}$ type II lines.

4. The pencil of a type II point consists of 1 type I line, $q^{4}$ type II lines and $q^{6}-q^{4}$ type III lines.

5. The pencil of a type III point consists of $q^{4}+q^{2}+1$ type II lines and $q^{6}-q^{4}-q^{2}$ type III lines.

6. $\quad$ There are $q^{4}+q^{2}+1$ type I points, $q^{10}+q^{8}-q^{4}-q^{2}$ type II points and $q^{12}-q^{10}-q^{8}+q^{6}$ type III points.

We assume the dual version of Lemma 2.1 (and other results whenever applicable) for the lines. Note that the type II points on a type I line are in $\left(q^{6}-q^{2}\right) / 3$ 3-cycles under $\alpha$.

Every type III line is uniquely determined by a type III point $x$ as $x \cdot x^{\alpha}$. Indeed, given a type III line $L$, let $x=L \cap L^{\alpha^{2}}$. Then $x^{\alpha}=L^{\alpha} \cap L$ and $x^{\alpha^{2}}=L^{\alpha^{2}} \cap L^{\alpha}$. Thus $x$ is a type III point and $L=x \cdot x^{\alpha}$. The Figueroa plane is obtained by the introduction of a new incidence, called $\mathcal{F}$-incidence, between the set of points $\mathcal{P}$ and the set of lines $\mathcal{L}$ of $P G\left(2, q^{6}\right)$, so that (viewing a line as a point set) the remaining $q^{6}-q^{4}-q^{2}-2$ type III points on the type III line $L=x . x^{\alpha}$ are replaced by other type III points to form a new line. We recall the definitions [6]: Let $\mu$ be an involutory bijection between the points of type III and the lines of type III given as follows: if a point $x \in \mathcal{P}$ and a line $L \in \mathcal{L}$ are both of type III, then $x^{\mu}=x^{\alpha} \cdot x^{\alpha^{2}}$, and $L^{\mu}=L^{\alpha} \cap L^{\alpha^{2}}$. The $\mathcal{F}$-incidence is defined as follows: if $x \in \mathcal{P}$ and $L \in \mathcal{L}$ are both of type III, then $x$ is $\mathcal{F}$-incident with $L$ if and only if $L^{\mu} \in x^{\mu}$ in $P G\left(2, q^{6}\right)$. In all other cases, $x$ is $\mathcal{F}$-incident with $L$ if and only if $x \in L$ in $P G\left(2, q^{6}\right)$. The (non-Desarguesian) projective plane obtained is the Figueroa plane of order $q^{6}$ which we denote by $F i g\left(q^{6}\right)$ (see $\left.[5,6,8]\right)$.

As a set of points, a type I line or a type II line in $P G\left(2, q^{6}\right)$ remains unchanged as a line in $F i g\left(q^{6}\right)$. As for a type III line in $P G\left(2, q^{6}\right)$, it becomes "twisted" in Fig $\left(q^{6}\right)$. More precisely, let $L$ be the type III line given by $x . x^{\alpha}$ where $x$ is a type III point. Let $y_{i}, i=1, \ldots, q^{6}-q^{4}-q^{2}-2$ be the remaining type III points on $L$. Consider the pencil of type III lines on the type III point $x^{\alpha^{2}}$. Other than $x^{\alpha^{2}} . x$ and $x^{\alpha^{2}} . x^{\alpha}$, the remaining type III lines in the pencil are given by $z_{i} . z_{i}^{\alpha}, i=1, \ldots, q^{6}-q^{4}-q^{2}-2$, where each $z_{i}$ is a type III point. 
Let $L_{\mathcal{F}}$ be the set of points obtained from $L$ by replacing each $y_{i}$ with $z_{i}^{\alpha^{2}}$. Then by the definition of $\mathcal{F}$-incidence, $L_{\mathcal{F}}$ is the Figueroa line corresponding to the type III line $L$. For some interesting results in this direction (which are not needed here) we refer readers to the work of Brown [1].

\section{Polar unital $\mathcal{U}$ in $\operatorname{Fig}\left(q^{6}\right)$}

Let $\rho$ be a unitary polarity of $P G\left(2, q^{6}\right)$ that commutes with $\alpha$. Then $\rho$ preserves types of points and lines of $P G\left(2, q^{6}\right)$. Let $\mathcal{H}$ be the classical unital defined by $\rho$, i.e. the unitary block design whose points are the absolute points of $\rho$ and whose blocks are the non-absolute lines of $\rho$. Again, since $\rho$ commutes with $\alpha, \mathcal{H}$ is preserved by $\alpha$. It is shown in [4] that $\rho$ induces a unitary polarity in Fig $\left(q^{6}\right)$ which thus defines a unital in Fig $\left(q^{6}\right)$. Let us denote the induced polarity by $\rho_{\mathcal{F}}$. We recall the construction: For points and lines of type I or type II, $\rho_{\mathcal{F}}=\rho$. For a type III point $x, x^{\rho_{\mathcal{F}}}=\left(x^{\rho}\right)_{\mathcal{F}}$, where $\left(x^{\rho}\right)_{\mathcal{F}}$ is the line in Fig $\left(q^{6}\right)$ corresponding to the type III line $x^{\rho}$ as described in Sect. 2. For a type III line $L,\left(L_{\mathcal{F}}\right)^{\rho_{\mathcal{F}}}=L^{\rho}$. Since $\rho$ commutes with $\mu, \rho_{\mathcal{F}}$ is indeed a polarity of $\operatorname{Fig}\left(q^{6}\right)$. Furthermore, if $x$ is a type III point, then $x$ is $\rho_{\mathcal{F}}$-absolute if and only if $x^{\mu \rho} \in \mathcal{H}$. Since $\mu \rho$ is a bijection, the number of type III points which are $\rho_{\mathcal{F}}$-absolute equals the number of type III points which are $\rho$-absolute. The number of absolute points of $\rho_{\mathcal{F}}$ is thus the same as that of $\rho$, so that $\rho_{\mathcal{F}}$ is unitary (a result of Seib [11]). We denote by $\mathcal{U}$ the unital defined by $\rho_{\mathcal{F}}$, and refer to it as the Figueroa unital induced by $\mathcal{H}$.

We have seen above that $\mathcal{H}$ and $\mathcal{U}$ share the same type I and type II points and have the same number of type III points. The following lemma shows that the type III points of $\mathcal{H}$ are distinct from those of $\mathcal{U}$.

Lemma 3.1. The set of type III points of $\mathcal{H}$ and the set of type III points of $\mathcal{U}$ are disjoint.

Proof. Let $x$ be a type III point. If $x$ is a point of $\mathcal{U}$, then $x \in x^{\rho_{\mathcal{F}}}=\left(x^{\rho}\right)_{\mathcal{F}}$, i.e. $x^{\rho \mu} \in x^{\mu}$ by definition of $\mathcal{F}$-incidence. Since $\rho$ commutes with $\mu$, this means that $x^{\mu \rho} \in x^{\mu}$, i.e. $x^{\mu}$ is $\rho$-absolute. On the other hand, if $x$ is a point of $\mathcal{H}$, then both $x^{\alpha}$ and $x^{\alpha^{2}}$ are points of $\mathcal{H}$, since $\rho$ commutes with $\alpha$ so that $\alpha$ preserves $\mathcal{H}$. Now by definition, $x^{\mu}=x^{\alpha} \cdot x^{\alpha^{2}}$, so $x^{\mu}$ is not $\rho$-absolute. This proves the lemma.

The following is the corresponding result for lines.

Lemma 3.2. Let $L$ be a type III line. If $L_{\mathcal{F}}$ is $\rho_{\mathcal{F}}$-absolute, then $L$ is $\rho$-nonabsolute.

Proof Let $L=x . x^{\alpha}$ where $x$ is of type III, as described in Sect. 2. If $L$ is $\rho$-absolute, then $L^{\rho}$ is the only $\rho$-absolute point on $L$, and so it can neither be $x$ nor $x^{\alpha}$ (as $\alpha$ preserves $\mathcal{H}$ ). On the other hand, if $L_{\mathcal{F}}$ is $\rho_{\mathcal{F}}$-absolute, then the type III point $L^{\rho}$ is in $L_{\mathcal{F}}$ by definition, and so it is either $x$ or $x^{\alpha}$, according to the structure of $L_{\mathcal{F}}$ as described in Sect. 2. This proves the lemma. 
The following corollary will be used in Sect. 4 to give the structure of certain type III lines of $\mathcal{H}$.

Corollary 3.3. Let $L$ be a type III line. If $L_{\mathcal{F}}$ is $\rho_{\mathcal{F}}$-absolute, then all the $\rho$-absolute points on $L$ are of type III.

Proof. By Lemma 3.2, $L$ is a non-absolute line of $\rho$. If $L$ had a type II $\rho$-absolute-point, then this point is also a $\rho_{\mathcal{F}}$-absolute point. On the other hand, the unique $\rho_{\mathcal{F}}$-absolute point on $L_{F}$ is $L^{\rho}$, which is of type III. This is a contradiction.

\section{Structure of classical unital $\mathcal{H}$ in $\operatorname{PG}\left(2, q^{6}\right)$ with respect to types}

In this section we study the structure of $\mathcal{H}$ with respect to types and prepare some lemmas to be used in the next section for the proof of our main result. We assume standard results in Galois geometry which can be found in [7] or [9].

Recall the unitary polarity $\rho$ of $P G\left(2, q^{6}\right)$ in Sect. 3 which commutes with the planar collineation $\alpha$ of $P G\left(2, q^{6}\right)$ in Sect. 2. Consider the classical unital $\mathcal{H}$ defined by $\rho$, and the subplane $\mathcal{P}_{\alpha}$ which constitutes the fixed elements of $\alpha$. Recall that $\mathcal{H}$ is preserved by $\alpha$ and that $\mathcal{P}_{\alpha}$ is isomorphic to $P G\left(2, q^{2}\right)$. We first note that $\mathcal{H}$ restricts to a classical unital in $\mathcal{P}_{\alpha}$ :

Lemma 4.1. The restriction of $\rho$ to $\mathcal{P}_{\alpha}$ is a unitary polarity.

Proof. Since $\rho$ preserves types and $\mathcal{P}_{\alpha}$ consists of the points and lines of type I, $\left.\rho\right|_{\mathcal{P}_{\alpha}}$ is a polarity of $\mathcal{P}_{\alpha}$. Since $\mathcal{P}_{\alpha} \cong P G\left(2, q^{2}\right),\left.\rho\right|_{\mathcal{P}_{\alpha}}$ is either unitary or orthogonal.

Suppose $\left.\rho\right|_{\mathcal{P}_{\alpha}}$ is orthogonal. We wish to obtain a contradiction. When $q$ is even, the $q^{2}+1$ absolute points of $\left.\rho\right|_{\mathcal{P}_{\alpha}}$ lie on a line, say $L$. Let $M$ be a type I non-absolute line of $\rho$ different from $L$. Then among the $q^{3}+1$ absolute points of $\rho$ on $M$, only one is of type I, namely, $M \cap L$, with the rest of type II. Since $\alpha$ preserves $M$ as well as $\mathcal{H}$, these $q^{3}$ points are in 3-cycles of $\alpha$. This is impossible as $q$ is an even prime power. When $q$ is odd, the absolute points of $\left.\rho\right|_{\mathcal{P}_{\alpha}}$ form an oval $\mathcal{O}$ in $\mathcal{P}_{\alpha}$. Let $L_{0}$ be a type I line external to $\mathcal{O}$ and $L_{2}$ a type I line secant to $\mathcal{O}$. Since a type I absolute line of $\rho$ is on a unique type I absolute point of $\rho$, both $L_{0}$ and $L_{2}$ are both non-absolute lines of $\rho$. As before, this requires $q^{3}+1 \equiv 0(\bmod 3)$ and $q^{3}-1 \equiv 0(\bmod 3)$ simultaneously, which is impossible.

Since $\mathcal{P}_{\alpha}$ is isomorphic to $P G\left(2, q^{2}\right),\left.\rho\right|_{\mathcal{P}_{\alpha}}$ defines a classical unital $\mathcal{H}_{\alpha}$ in $\mathcal{P}_{\alpha}$. $\mathcal{H}_{\alpha}$ is thus the restriction of $\mathcal{H}$ to $\mathcal{P}_{\alpha}$. The following theorem can now be easily verified.

Theorem 4.2. 1. A type I line of $\mathcal{H}$ is incident with $q+1$ type I points of $\mathcal{H}$ and $q^{3}-q$ type II points of $\mathcal{H}$.

2. Through a type I point of $\mathcal{H}$, there are $q^{2}$ type I lines of $\mathcal{H}$ and $q^{6}-q^{2}$ type II lines of $\mathcal{H}$. 
3. Through a type I point which is not of $\mathcal{H}$, there are $q^{2}-q$ type I lines of $\mathcal{H}$ and $q^{6}-q^{3}-q^{2}+q$ type II lines of $\mathcal{H}$.

4. The set of points of $\mathcal{H}$ consists of $q^{3}+1$ points of type $I, q^{7}-q^{6}+q^{4}-q^{3}$ of type II and $q^{9}-q^{7}+q^{6}-q^{4}$ of type III. The set of lines of $\mathcal{H}$ consists of $q^{4}-q^{3}+q^{2}$ lines of type $I, q^{10}+q^{8}-q^{7}+q^{6}-2 q^{4}+q^{3}-q^{2}$ of type II and $q^{12}-q^{10}-q^{9}-q^{8}+q^{7}+q^{4}$ of type III.

Proof. The first three parts are immediate from Lemma 4.1. We illustrate the counting in part (4). Again, since $\mathcal{H}_{\alpha}$ is the restriction of $\mathcal{H}$ to $\mathcal{P}_{\alpha}, \mathcal{H}$ has $q^{3}+1$ type I points and $q^{4}-q^{3}+q^{2}$ type I lines. Then by (1) and Lemma 2.1 (1), the number of type II points of $\mathcal{H}$ is given by number of type II points of $\mathcal{H}$ on the type I lines of $\mathcal{H}$, i.e. $\left(q^{3}-q\right)\left(q^{4}-q^{3}+q^{2}\right)=q^{7}-q^{6}+q^{4}-q^{3}$. The remaining $\left(q^{9}+1\right)-\left(q^{7}-q^{6}+q^{4}-q^{3}\right)-\left(q^{3}+1\right)=q^{9}-q^{7}+q^{6}-q^{4}$ points of $\mathcal{H}$ are of type III. By (2), (3) and the dual of Lemma 2.1 (1), the number of type II lines of $\mathcal{H}$ is given by the number of such lines through points of $\mathcal{H}$ and points not of $\mathcal{H}$, i.e. $\left(q^{3}+1\right)\left(q^{6}-q^{2}\right)+\left(q^{4}-q^{3}+q^{2}\right)\left(q^{6}-q^{3}-q^{2}+q\right)=q^{10}+q^{8}-q^{7}+q^{6}-2 q^{4}+q^{3}-q^{2}$. The remaining $\left(q^{12}-q^{9}+q^{6}\right)-\left(q^{4}-q^{3}+q^{2}\right)-\left(q^{10}+q^{8}-q^{7}+q^{6}-2 q^{4}+q^{3}-q^{2}\right)=$ $q^{12}-q^{10}-q^{9}-q^{8}+q^{7}+q^{4}$ lines of $\mathcal{H}$ are of type III.

We next study the structure of the type II lines of $\mathcal{H}$. We shall need two fundamental results. The first is the non-existence of the O'Nan configuration (four unital lines intersecting in six unital points) in a classical unital [10]. The second is a characterization of the extrinsic relation of perpendicularity between lines of a classical unital embedded in the classical plane in terms only of the incidence structure of the unitary block design [12].

Let $a$ be a type I absolute point and $x$ a type I non-absolute point on $a^{\rho}$. By Theorem 3.3 of [12] (a synthetic proof of which is given in [2] and in which a superfluous hypothesis in [12] is removed) applied to $\mathcal{H}$, the set of non-absolute lines through $x$ can be partitioned into $q^{3}-1$ subsets $\mathcal{M}_{1}, \ldots, \mathcal{M}_{q^{3}-1}$, each of cardinality $q^{3}$, and the set of non-absolute lines on $a$, except $x^{\rho}$, can be partitioned into $q^{3}-1$ subsets $\mathcal{N}_{1}, \ldots, \mathcal{N}_{q^{3}-1}$, each of cardinality $q^{3}+1$, such that if $M \in \mathcal{M}_{i}$ and $N \in \mathcal{N}_{j}$, then $M$ and $N$ meet at an absolute point if and only if $i=j$.

Now let $M$ be a type I non-absolute line on $x$. Then it is in $\mathcal{M}_{i}$ for some $i \in\left\{1, \ldots, q^{3}-1\right\}$. Write $\mathcal{M}_{i}=\mathcal{M}$ and denote by $\mathcal{M}_{I}$ and $\mathcal{M}_{I I}$ respectively the sets of type I and type II lines of $\mathcal{M}$. Similarly, write $\mathcal{N}_{i}=\mathcal{N}$ and denote by $\mathcal{N}_{I}$ and $\mathcal{N}_{I I}$ respectively the sets of type I and type II lines of $\mathcal{N}$. We have the following lemma:

Lemma 4.3. 1. $\left|\mathcal{M}_{I}\right|=q,\left|\mathcal{M}_{I I}\right|=q^{3}-q,\left|\mathcal{N}_{I}\right|=q+1,\left|\mathcal{N}_{I I}\right|=q^{3}-q$.

2. Lines of $\mathcal{N}_{I}$ and lines of $\mathcal{M}_{I I}$ meet in points of type II.

3. Lines of $\mathcal{N}_{I I}$ and lines of $\mathcal{M}_{I}$ meet in points of type II.

4. Lines of $\mathcal{N}_{I I}$ and lines of $\mathcal{M}_{I I}$ meet in points of type III.

Proof. The first three parts are easy consequences of Theorem 4.2. We prove part (4). Let $M_{2} \in \mathcal{M}_{I I}, N_{1} \in \mathcal{N}_{I}, N_{2} \in \mathcal{N}_{I I}, b_{1}=M_{2} \cap N_{1}$ and $b_{2}=M_{2} \cap N_{2}$. We wish to show that $b_{2}$ is of type III. First note that by the dual of Lemma 2.1 


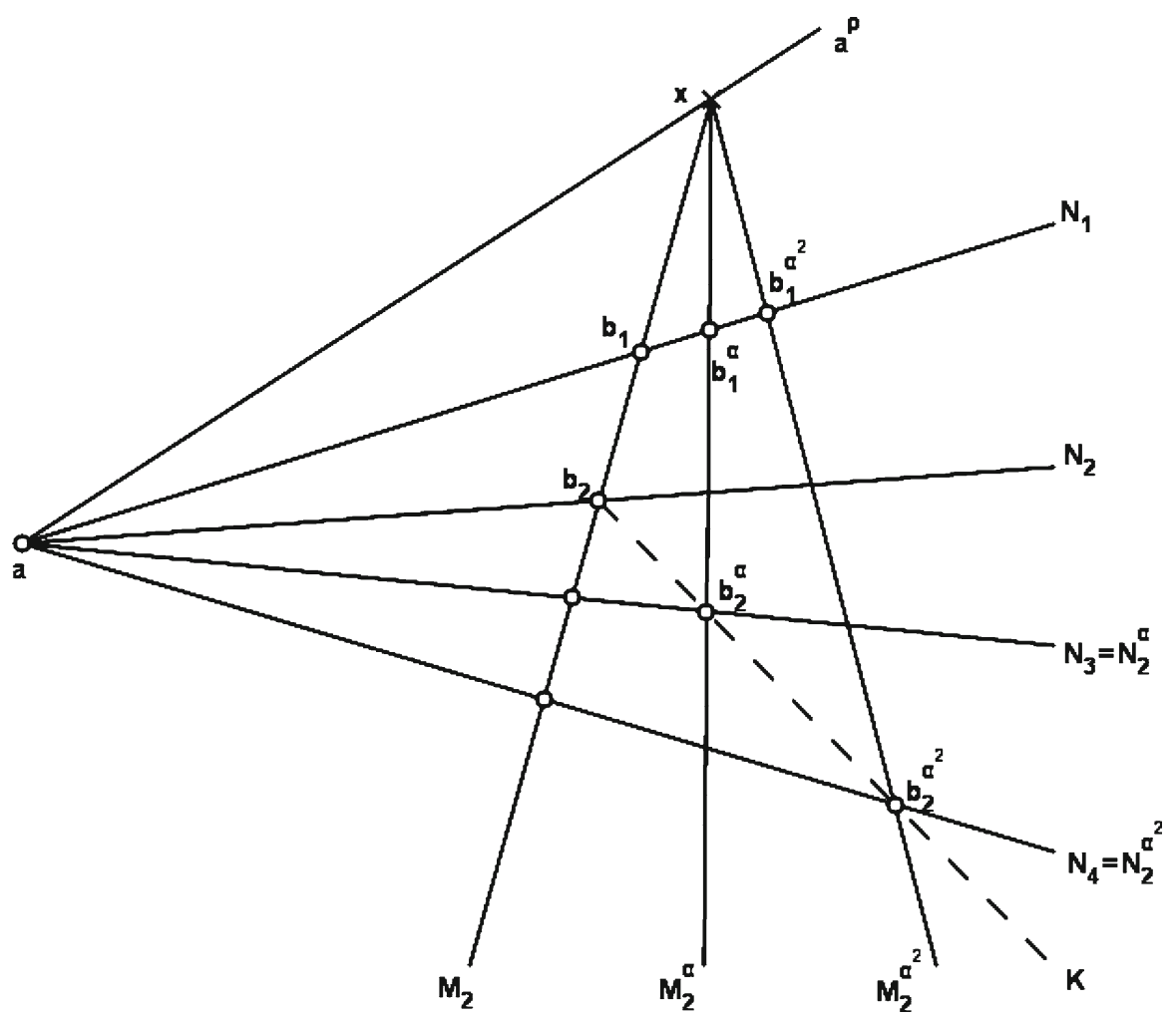

Figure 1 O'Nan configuration in the proof of Lemma 4.3 (4)

(1), $b_{2}$ is not of type I as it is on the type II line $M_{2}$ and the unique type I point on $M_{2}$ is the point $x$. Next suppose $b_{2}$ is a type II point. Then $b_{2}, b_{2}^{\alpha}$ and $b_{2}^{\alpha^{2}}$ are on a type I line, say $K$, by Lemma 2.1 (1). On the other hand, since $b_{2}$ is on $M_{2}=b_{1} \cdot x, b_{2}^{\alpha}$ and $b_{2}^{\alpha^{2}}$ are respectively on $M_{2}^{\alpha}=b_{1}^{\alpha} \cdot x$ and $M_{2}^{\alpha^{2}}=b_{1}^{\alpha^{2}} \cdot x$, which are lines of $\mathcal{M}_{I I}$ as $M_{2}^{\alpha}$ and $M_{2}^{\alpha^{2}}$ pass through $x$ and the orbit of $b_{1}$ is on $N_{1}$. It follows that $b_{2}^{\alpha}$ and $b_{2}^{\alpha^{2}}$ are respectively on some lines, say $N_{3}$ and $N_{4}$, of $\mathcal{N}_{I I}$. Note that $N_{3}=N_{2}^{\alpha}$ and $N_{4}=N_{2}^{\alpha^{2}}$. Then the six points $a, b_{2}, b_{2}^{\alpha}$, $b_{2}^{\alpha^{2}}, M_{2} \cap N_{3}, M_{2} \cap N_{4}$ and the four lines $M_{2}, K, N_{3}$ and $N_{4}$ form an O'Nan configuration in $\mathcal{H}$. See Fig. 1 . This is impossible by [10]. Hence $b_{2}$ is of type III, as we wished.

We are now ready to deduce the following partial result on the structure of type II lines of $\mathcal{H}$ with respect to types.

Theorem 4.4. 1. Among the type II lines through a type I absolute point, there are $q^{2}(q-1)\left(q^{3}-q\right)$ lines each of which is incident with exactly one type I, $q$ type II and $q^{3}-q$ type III absolute points. The remaining $q^{2}(q-1)(q+1)^{2}$ lines are each incident with one type $I$ and $q^{3}$ type III absolute points. 
2. There are $\left(q^{3}+1\right) q^{2}(q-1)\left(q^{3}-q\right)$ type II lines each of which is incident with one type I, q type II and $q^{3}-q$ type III absolute points, and there are $\left(q^{3}+1\right) q^{2}(q-1)(q+1)^{2}$ type II lines each of which is incident with one type $I$ and $q^{3}$ type III absolute points.

3. Among the type II lines through a type I non-absolute point, there are at least $(q+1)(q-1)\left(q^{3}-q\right)$ lines each of which is incident with $q+1$ type II and $q^{3}-q$ type III absolute points.

4. There are at least $\left(q^{4}-q^{3}+q^{2}\right)(q+1)(q-1)\left(q^{3}-q\right)$ lines each of which is incident with $q+1$ type II and $q^{3}-q$ type III absolute points.

\section{Proof.}

(1) Let $a$ be a type I absolute point. To study the number of absolute points of each type on a type II line through $a$, it suffices to count the number of type II absolute points on it. Note that by Lemma 2.1 (1), if $b$ is a point on a type II line through $a$, then $b$ is of type II if and only if it is on a type I line not through $a$. Therefore we study how type I lines not through $a$ meet the type II pencil of $a$.

Let $x_{1}, x_{2}, \ldots, x_{q^{2}}$ be the type I non-absolute points on $a^{\rho}$. For $i=1,2, \ldots, q^{2}$, let $\mathfrak{M}_{i}$ be the set of non-absolute lines though $x_{i}$.

Consider $\mathfrak{M}_{1}$. Repeating the argument in Lemma 4.3 (1), we obtain $q-1$ disjoint subsets $\mathcal{M}_{1}, \ldots, \mathcal{M}_{q-1}$ of $\mathfrak{M}_{1}$ such that each subset contains $q$ type I lines and $q^{3}-q$ type II lines, and every line of each subset meets $q+1$ type I lines through $a$ and $q^{3}-q$ type II lines through $a$ at absolute points. Let $\mathfrak{N}_{1}$ be the set of these $(q-1)\left(q^{3}-q\right)$ type II lines through $a$. By Lemma 4.3, these $(q-1)\left(q^{3}-q\right)$ type II lines are distinct as no line can meet lines of more than one subset among $\mathcal{M}_{1}, \ldots, \mathcal{M}_{q-1}$ at absolute points, and each of these lines contains $q$ type II absolute points and $q^{3}-q$ type III absolute points.

Similarly, for $i=2,3, \ldots, q^{2}$, by considering $\mathfrak{M}_{i}$, we obtain $\mathfrak{N}_{i}$. We claim that the $\mathfrak{N}_{i}$ 's are disjoint. If the claim is true, then there are $\left|\bigcup_{i=1}^{q^{2}} \mathfrak{N}_{i}\right|=$ $q^{2}(q-1)\left(q^{3}-q\right)$ type II lines through $a$ having $q$ type II absolute points and $q^{3}-q$ type III absolute points on each line. Since $\bigcup_{i=1}^{q^{2}} \mathfrak{N}_{i}$ contains all the lines through $a$ that meet some type I non-absolute lines not through $a$ at absolute points, each of the remaining $\left(q^{6}-q^{2}\right)-q^{2}(q-1)\left(q^{3}-q\right)=q^{2}(q-1)(q+1)^{2}$ type II lines through a contains no type II absolute point, but one type I and $q^{3}+1$ type III absolute points.

To prove our claim, suppose there is a line $N$ belonging to both $\mathfrak{N}_{1}$ and $\mathfrak{N}_{2}$. Since $N$ is of type II, $N$ meets some type I line $M_{1}$ through $x_{1}$ at some type II absolute point $b$ by Lemma 4.3. Since $N \in \mathfrak{N}_{2}$ and $b$ is of type II, by Lemma 4.3 again, there is a type I line $M_{2} \in \mathfrak{M}_{2}$ such that $b \in M_{2}$. Then $b$ is on two distinct type I lines, contradicting Lemma 2.1 (1).

(2) This follows from (1) since there are $q^{3}+1$ type I absolute points, by Theorem 4.2 (4). 
(3) Similar to (1).

(4) Similar to (2).

The following is a partial result on the structure of type III lines of $\mathcal{H}$ with respect to types:

Theorem 4.5. There are at least $q^{9}-q^{7}+q^{6}-q^{4}$ type III non-absolute lines each of which contains absolute points of type III only.

Proof. The number of type III lines $L$ such that $L_{\mathcal{F}}$ is $\rho_{\mathcal{F}}$-absolute equals the number of type III points which are $\rho_{\mathcal{F}}$-absolute, and we have seen in Sect. 3 that this is the same as the number of type III $\rho$-absolute points, which is $q^{9}-q^{7}+q^{6}-q^{4}$ by Theorem 4.2 (4). Now apply Corollary 3.3.

Remark 4.6. In a Figueroa plane of specific order it should not be too difficult to show (and for small orders it is known) that there exist O'Nan configurations. However, it is not known if this is true in general. Structure theorems such as those obtained above may play a role in an attempt to settle the question, and work by the authors in this direction is in progress.

\section{The Figueroa unital $\mathcal{U}$ is not classical}

In this section we prove that $\mathcal{U}$ is not classical. Since a classical unital satisfies Wilbrink's Condition (II) [13], it suffices to show that this condition does not hold in $\mathcal{U}$.

Lemma 5.1. $\mathcal{U}$ does not satisfy Condition $(I I)$.

Proof. Suppose $\mathcal{U}$ satisfies Condition $(I I)$. We consider the following special case. Let $a$ be a type I point of $\mathcal{U}$ on a type I line $L$ of $\mathcal{U}$. Let $M$ be a line of $\mathcal{U}$ meeting $L$ at a type I point $b$ of $\mathcal{U}$ different from $a$ and meeting $a^{\rho_{\mathcal{F}}}$ at a type I non-absolute point $x$. Note that $M$ is thus a type I line not incident with $a$. Let $\mathcal{L}_{I}$ and $\mathcal{L}_{I I}$ respectively be the sets of type I and type II lines of $\mathcal{U}$ through $a$ which meet $M$. (Note that as $a$ is a type I points, there are no type III lines of Fig $\left(q^{6}\right)$ on it.) Let $b^{\prime}$ be a type II point on $L$. By Condition $(I I)$, there exists a line $M^{\prime}$ of $\mathcal{U}$ passing through $b^{\prime}$ but not $a$ and intersecting all lines from $a$ which meet $M$. We claim that $M^{\prime}$ is incident with $x$.

Suppose the contrary. Thus $M^{\prime}$ and $x \cdot b^{\prime}$ are different lines, and since $a \cdot b^{\prime}$ is type I and $b^{\prime}$ is on $M^{\prime}, M^{\prime}$ is of type II or III, by Lemma 2.1 (1). Suppose $M^{\prime}$ is of type II. Then $a, b, b^{\prime}, L, M, M^{\prime}, x \cdot b^{\prime}$ and lines of $\mathcal{L}_{I}$ and $\mathcal{L}_{I I}$ are points or lines of $\mathcal{H}$, as they are all of type I or II only. By Theorem 3.3 of [12] (or Lemma 2.1 of [2]), x. $b^{\prime}$ meets every line of $\mathcal{L}_{I}$ at a point of $\mathcal{H}$ since this is true for $x . b$. Now both $x . b^{\prime}$ and $M^{\prime}$ meet every line of $\mathcal{L}_{I}$ at a point of $\mathcal{H}$. It follows that $x . b^{\prime}$ and $M^{\prime}$, together with any two lines of $\mathcal{L}_{I} \backslash\{L\}$, form an O'Nan configuration in $\mathcal{H}$. This is impossible. Next suppose $M^{\prime}$ is of type III. Since lines of $\mathcal{L}_{I}$ are of type I and so they contain no type III points, the intersection point of $M^{\prime}$ and any line of $\mathcal{L}_{I}$ is of type I or II only. We thus obtain the same contradiction in the same manner. 
We have shown that $M^{\prime}$ is the line $x \cdot b^{\prime}$. So $M^{\prime}$ is not of type III, by the dual of Lemma $2.1(2)$. It is not of type I since it meets the type I line $a . b^{\prime}$ at the type II point $b^{\prime}$. Hence $M^{\prime}$ is of type II. By Lemma $4.3(4), M^{\prime}$ meets every line of $\mathcal{L}_{I I}$ at a type III absolute point of $\mathcal{H}$. By Lemma 3.1, such a point is never a point of $\mathcal{U}$. We have a contradiction.

The following result is now immediate from Lemma 5.1:

Theorem 5.2. The Figueroa unital $\mathcal{U}$ is not classical.

Open Access. This article is distributed under the terms of the Creative Commons Attribution License which permits any use, distribution, and reproduction in any medium, provided the original author(s) and the source are credited.

\section{References}

[1] Brown, J.M.N.: Some partitions in Figueroa planes. Note Math. 29, 33-44 (2009)

[2] Chan, K.Y., Hui, M.W., Wong, P.P.W.: A synthetic proof of a theorem of Taylor on perpendicularity in a classical unital. Manuscript (2011)

[3] Dembowski, P.: Finite Geometries. Springer, Berlin (1968)

[4] de Resmini, M.J., Hamilton, N.: Hyperovals and unitals in Figueroa planes. Eur. J. Combin. 19, 215-220 (1998)

[5] Figueroa, R.: A family of not $(V, l)$-transitive projective planes of order $q^{3}, q \not \equiv 1$ $(\bmod 3)$ and $q>2$. Math. Z. 181, 471-479 (1982)

[6] Grundhöfer, T.: A synthetic construction of the Figueroa planes. J. Geom. 26, 191-201 (1986)

[7] Hirschfeld, J.W.P.: Projective geometries over finite fields, 2nd edn. Clarendon Press, Oxford (1998)

[8] Hering, Ch., Schaeffer, H.J.: On the new projective planes of R. Figueroa. In: Jungnickel, D. et al. (eds.) Combinatorial Theory. Proc. Schloss Rauischholzhausen 1982, pp. 187-190. Springer, Berlin (1982)

[9] Hughes, D.R., Piper, F.C.: Projective Planes. Springer, New York (1973)

[10] O'Nan, M.E.: Automorphisms of unitary block designs. J. Algebra 20, 495511 (1971)

[11] Seib, M.: Unitäre Polaritäten endlicher projectiver Ebenen. Arch. Math. (Basel) 21, 103-112 (1970)

[12] Taylor, D.E.: Unitary Block designs. J. Comb. Theory Ser. A. 16, 51-56 (1974)

[13] Wilbrink H.: A characterization of the classical unitals. In: Johnson, N.L. et al. (eds.) Finite Geometries. Lecture Notes Pure Appl. Math., vol. 82, pp 445-454. Dekker, New York (1983)

Man Wa Hui

Department of Mathematics

University of Hong Kong

Hong Kong, China

e-mail: huimanwa@gmail.com 
Philip P. W. Wong

Department of Mathematics

University of Hong Kong

Hong Kong, China

e-mail: ppwwong@maths.hku.hk

Received: January 31, 2012.

Revised: July 16, 2012. 